\title{
ÖGIM FLIP
}

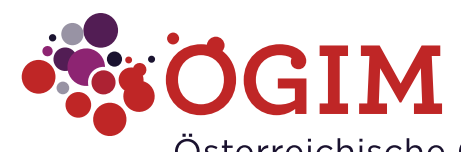

Österreichische Gesellschaft für Innere Medizin

(c) Springer-Verlag Wien 2013
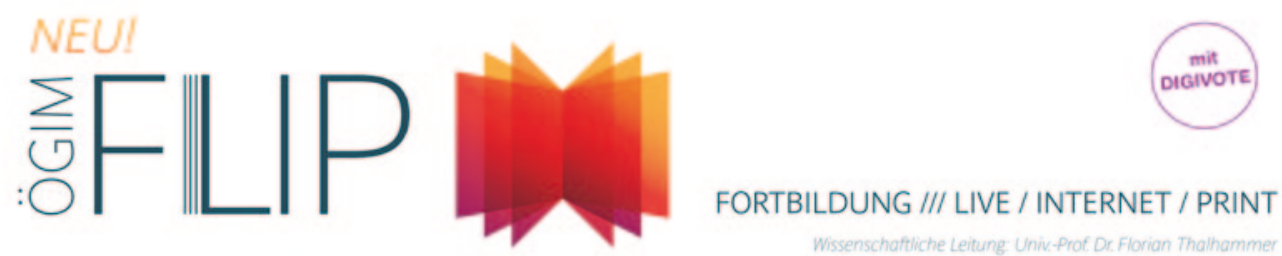

Österreichs modernstes internistisches

Fortbildungsformat für alle fertigen

bzw. in Ausbildung stehenden Fachärztınnen.

Montag, 11. März 2013, 17.00-20.15 Herz Vorhofflimmern

Endokarditis

MD Rhuthmustörunoes

Univ-Prof Dr Helmut Piverfelliner Limy

Univ-Prof. De Martin Hulsmann, Wen

proverang

Montag 22. April 2013, 17.00-20.15

Lunge

Asthma und COPC

Bronchuskatrinom
DD Akuter Thorarschmert

Infektionen des Respirationstrakts

Univ-Doz Dr.Arschang valipour Wien
Univ-Prof Dr Sahine zochhouer-Miller Wien

Drei Lernmioglichlesten

Live / Internet / Print

Montag, 10. Juni 2013, 17.00-20.15

Niere

Anterielle Hypertonie

Nierenzellkarzinom

Zystris und Pyelonephritis

$$
\text { Uni }
$$

Dr Peter Smetana, Wis

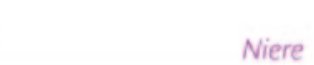

$\bullet$

-

Montag, 23. September 2013, 17.00-20.15 Darm

Univ-Prof. Dr. Alexander Rosenkranz, Gra

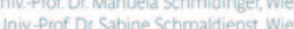
Univ-Prof Dr. Florian Thathammer, Wies

Antibiotika-assozierte Diarthoe
DD Akves Abdomen

DD Akutes Abdomen
(inkl. Familiares Mittelmeerfieber $=$ FMW)

Kolonkarzinom

Colitis ulcerosa und Morbus Crohn

Univ- Prof Dr Werner Scheithauer Wien

Montag, 21. Oktober 2013, 17.00-20.15

Blut

DD Anämie

Leukamien

(Malaria, Babesiose, Trypanosomiasis)

Moderne Antikoagulationsoptionen

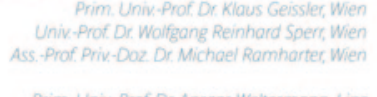

Montag 11. November 2013, 17.00-20.15

Leber

Hepatitis A bis E

DD Cholestase

Hepatozellulates Karzinom

Leberzirthose

Informationen und kostenlose Anmeldung: www.oegim-flip.at

Raiffeisen-Forum Wien, Friedrich-Wilhelm-Raiffeisen-Platz 1, 1020 Wier

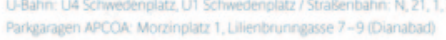

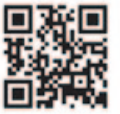

MEDIZIN MEDIEN AUSTRIA

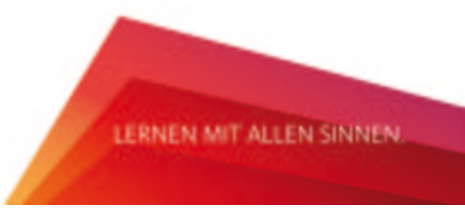

